\title{
Interação genótipo $x$ ambiente em genótipos de feijão-caupi semiprostrado via modelos mistos
}

\author{
Francisco Eduardo Torres ('); Paulo Eduardo Teodoro ( $\left.{ }^{1 *}\right)$; Edvaldo Sagrilo ( $\left.{ }^{2}\right)$; Gessi Ceccon (3); \\ Agenor Martinho Correa (') \\ (') Universidade Estadual do Mato Grosso do Sul (UEMS), Departamento de Fitotecnia, \\ Rodovia Aquidauana, km 12, 79200-000 Aquidauana (MS), Brasil. \\ (2) Embrapa Meio-Norte, Avenida Duque de Caxias, 5650, 64006-220 Teresina (PI), Brasil. \\ (3) Embrapa Agropecuária Oeste, Caixa Postal 661, 79804-970 Dourados (MS), Brasil. \\ (*) Autor correspondente: eduteodoro@hotmail.com
}

Recebido: 10/mar./2015; Aceito: 17/abr./2015

\begin{abstract}
Resumo
A metodologia de modelos mistos (REML/BLUP) tem sido empregada para estudar os efeitos da interação genótipo x ambiente (G x E) em várias culturas, como: arroz, feijão, cana-de-açúcar, cajueiro e eucalipto, porém ainda não foi aplicada em feijão-caupi. Assim, o objetivo deste trabalho foi selecionar simultaneamente genótipos de feijão-caupi semiprostrado cultivados no Estado do Mato Grosso do Sul, via modelos mistos, que reúnam alta adaptabilidade, estabilidade e produtividade de grãos. Foram conduzidos quatro ensaios de valor de cultivo e uso com genótipos de genótipos de feijão-caupi nos anos de 2005 e 2006 em Aquidauana, Chapadão do Sul e Dourados. O delineamento experimental utilizado foi o de blocos completos casualizados, com 20 genótipos e 4 repetições. Os parâmetros genéticos foram estimados pela metodologia REML/BLUP, e a seleção baseou-se no método da média harmônica do desempenho relativo dos valores genéticos (MHPRVG), em três estratégias: seleção com base no valor genético predito, tendo-se considerado o desempenho médio dos genótipos em todos os ambientes (sem efeito de interação) ou o desempenho em cada ambiente (com efeito da interação); e seleção simultânea quanto à produtividade de grãos, estabilidade e adaptabilidade. Os genótipos BRS Paraguaçu, MNC99-542F-5 e MNC99-508G-1 podem ser cultivados em vários ambientes, pois reúnem alta produtividade de grãos, adaptabilidade e estabilidade. A herdabilidade da média dos genótipos apresentou magnitude variando de moderada a alta, fato que indica excelentes possibilidades para a seleção, permitindo acurácia seletiva de $82 \%$.
\end{abstract}

Palavras-chave: BLUP/REML, parâmetros genéticos, Vigna unguiculata L.

\section{Genotype $x$ environment interaction in semiprostrade cowpea genotypes via mixed models}

\section{Abstract}

The mixed model methodology (REML / BLUP) has been used to study the effects of genotype $x$ environment interaction ( $\mathrm{x}$ E) in various crops, such as: rice, common bean, cane sugar, cashew and eucalyptus, but still was not applied in cowpea. The aim of this work was to select simultaneously semiprostrade cowpea genotypes grown in the state of Mato Grosso do Sul, via mixed models, bringing together high adaptability, stability and yield grain. It were conducted four growing amount of trials and use of genotypes of cowpea genotypes in 2005 and 2006 in Aquidauana, Chapadão do Sul and Dourados. The experimental design was a randomized complete blocks with four replications and 20 genotypes. The genetic parameters were estimated by REML/BLUP methodology and, the selection was based on the MHPRVG method (harmonic mean of the relative performance of genetic values) in three strategies: selection based on predicted breeding value, having considered the performance mean of genotypes in all environments (no interaction effect) or performance in each environment (with interaction effect); and simultaneous selection for grain yield, stability and adaptability. BRS Paraguaçu, MNC99-542F-5 and MNC99-508G-1 genotypes can be grown in various environments, as they bring together high grain yield, adaptability and stability. The heritability of the average of genotypes found indicates moderate to high, a fact which indicates excellent possibilities for selection, allowing selective accuracy of $82 \%$.

Key words: BLUP/REML, genetic parameters, Vigna unguiculata L. 


\section{INTRODUÇÃO}

O feijão caupi-caupi (Vigna unguiculata (L.) Walp.) é uma das fontes alimentares mais importantes e estratégicas para as regiōes tropicais e subtropicais do planeta. Atualmente, o Brasil é o terceiro maior produtor mundial de feijáo-caupi, sendo cultivado, sobretudo, nas regióes Norte e Nordeste, onde se constitui no prato básico das classes de menor poder aquisitivo (Santos et al., 2014a). Contudo, apesar da grande produção brasileira, há déficit permanente de oferta nestas regiōes, uma vez que a produtividade média brasileira é extremamente baixa (em torno de $300 \mathrm{~kg} \mathrm{ha}^{-1}$ ). Santos et al. (2014b) enfatizam que o melhoramento genético desta cultura é o principal modo de aumentar sua produtividade média, selecionando, por meio de ensaios de valor de cultivo e uso, genótipos altamente produtivos e adaptados às condiçóes edafoclimáticas brasileiras.

Nos programas de melhoramento de feijão-caupi, um grande número de genótipos é testado anualmente em diferentes ambientes, antes de sua recomendaçáo final e multiplicaçáo (Santos et al., 2014a). Uma vez que, na maioria das vezes, estes ambientes são distintos, há interação entre genótipo e ambiente ( $\mathrm{G} \times \mathrm{E})$, o que afeta o ganho com a seleção e torna necessário estimar a magnitude e a natureza dessa interação. Essas estimativas possibilitam a avaliação do real impacto de seleçâo e asseguram alto grau de confiabilidade na recomendação de genótipos para um determinado local ou grupo de ambientes (Rosado et al., 2012).

Entretanto, apesar de sua importância, a simples análise da interação $\mathrm{G} x$ E não proporciona informaçôes completas e exatas sobre o comportamento de cada genótipo em várias condiçóes ambientais. Faz-se necessário realizar análises de adaptabilidade e estabilidade fenotípica, pelas quais é possível a identificação de genótipos com comportamento previsível, que sejam responsivos às variaçôes ambientais, em condiçôes específicas ou amplas (Cruz et al., 2014). Dessa forma, metodologias de seleção que incorporam a estabilidade e a adaptabilidade em uma única estatística podem ser consideradas superiores, em comparação àquelas que usam apenas a produção como critério de seleção (Resende, 2007).

A análise realizada por meio da metodologia de modelos mistos, também chamada método REML/BLUP foi proposta para culturas perenes como o pinus (Resende et al., 1996) e sua aplicação tem se disseminado na análise de informaçóes de diversas culturas, tanto perenes como anuais. $\mathrm{O}$ procedimento REML (Restricted Maximum Likelihood), ou máxima verossimilhança restrita, estima componentes de variância necessários ao modelo e o BLUP (Best Linear Unbiased Prediction), melhor preditor linear não viesado, estima o valor genotípico (Resende, 2007).

A metodologia de modelos mistos permite considerar erros correlacionados dentro de locais, bem como a estabilidade e a adaptabilidade na seleção de genótipos superiores; fornece valores genéticos já descontados da instabilidade; e pode ser aplicado a qualquer número de ambientes. Além disso, ele gera resultados na própria unidade ou escala do caráter avaliado, que podem ser interpretados diretamente como valores genéticos, o que os outros métodos não permitem. Esse modelo também possibilita inferências adicionais, como: seleção de genótipos específicos para cada local; seleção de genótipos estáveis através dos locais; seleção de genótipos responsivos (com alta adaptabilidade) à melhoria do ambiente; e seleção pelos três atributos (produtividade, estabilidade e adaptabilidade), simultaneamente (Rosado et al., 2012).

As estatísticas média harmônica dos valores genéticos (MHVG), desempenho relativo dos valores genéticos (PRVG) e média harmônica do desempenho relativo dos valores genéticos (MHPRVG) preditos têm sido usadas como medidas para interpretação da estabilidade genotípica e da adaptabilidade de culturas como feijāo (Carbonell et al., 2007), cana-de-açúcar (Bastos et al., 2007), cajueiro (Maia et al., 2009), eucalipto (Rosado et al., 2012) e arroz (Regitano et al., 2013). Entretanto, não há na literatura nenhuma pesquisa que investigou a interação $\mathrm{G} \times \mathrm{E}$ em genótipos de feijāo-caupi, via modelos mistos. Assim, o objetivo deste trabalho foi selecionar simultaneamente genótipos de feijão-caupi semiprostrado cultivados no Estado do Mato Grosso do Sul, via modelos mistos, que reúnam alta adaptabilidade, estabilidade e produtividade de grãos.

\section{MATERIAL E MÉTODO}

Foram conduzidos quatro ensaios de valor de cultivo e uso com linhagens e cultivares de genótipos de feijão-caupi nos anos de 2005 e 2006, nos municípios de Aquidauana, Chapadão do Sul e Dourados, cujas características edafoclimáticas estão expressas na tabela 1. O delineamento experimental utilizado foi o de blocos completos casualizados, com 20 tratamentos e 4 repetiçóes. A unidade experimental foi constituída por quatro fileiras de 5,0 $\mathrm{m}$ de comprimento, espaçadas em $0,5 \mathrm{~m}$ entre fileiras e $0,25 \mathrm{~m}$ entre plantas dentro da fileira. Em cada unidade experimental, a produtividade de grãos (PROD) foi avaliada nas duas fileiras centrais, sendo corrigida para $13 \%$ de umidade e extrapolada para $\mathrm{kg} \mathrm{ha}^{-1}$.

Os tratamentos foram constituídos por 18 linhagens (MNC99-505G-11, MNC99-507G-4, MNC99-507G-8,

Tabela 1. Ambientes, número de genótipos, ano agrícola, local, latitude, longitude, altitude dos ensaios de valor de cultivo e uso com 20 genótipos de feijão-caupi de porte semiprostrado, avaliados em quatro ambientes no Estado do Mato Grosso do Sul

\begin{tabular}{cccccc|}
\hline Ambiente & Ano & Local & Latitude & Longitude & Altitude \\
\hline 1 & 2005 & Aquidauana & $22^{\circ} 01^{\prime} \mathrm{S}$ & $5^{\circ} 05^{\prime} \mathrm{W}$ & $430 \mathrm{~m}$ \\
\hline 2 & 2005 & Chapadão do Sul & $18^{\circ} 05^{\prime} \mathrm{S}$ & $52^{\circ} 04^{\prime} \mathrm{W}$ & $790 \mathrm{~m}$ \\
\hline 3 & 2006 & Aquidauana & $22^{\circ} 01^{\prime} \mathrm{S}$ & $5^{\circ} 05^{\prime} \mathrm{W}$ & $430 \mathrm{~m}$ \\
\hline 4 & 2006 & Dourados & $20^{\circ} 03^{\prime} \mathrm{S}$ & $5^{\circ} 05^{\prime} \mathrm{W}$ & $147 \mathrm{~m}$ \\
\hline
\end{tabular}


BRS Xiquexique, MNC99-510G-8, MNC99-510F-16, TE97-309G-18, TE97-304G-4, TE97-304G-12, TE97-309G-24, MNC99-508G-1, MNC99-541F-15, MNC99-541F-18, MNC99-541F-21, MNC99-542F-5, MNC99-542F-7, MNC99-547F-2 e CNC x 409-11F-P2) e duas cultivares (BRS Paraguaçu e BR 17-Gurgueia) de feijão-caupi oriundas do programa de melhoramento genético de feijão-caupi da Embrapa Meio-Norte. Assim, os efeitos de genótipos foram considerados como aleatórios, conforme Resende \& Duarte (2007), que recomendam tratar efeitos genotípicos como aleatórios quando o número de tratamentos for igual ou superior a 10 .

Para avaliar o efeito da interação $\mathrm{G} \times \mathrm{E}$, foi utilizado o modelo estatístico 54 do software Selegen-Reml/Blup (Resende, 2007), correspondente $\mathrm{a}=\mathrm{Xb}+\mathrm{Zg}+\mathrm{Wc}+\mathrm{e}$, em que $\mathrm{y}, \mathrm{b}, \mathrm{g}$, c, e correspondem, respectivamente, aos vetores de dados de efeitos fixos (médias de blocos através dos ambientes), efeitos dos genótipos (aleatório), efeitos da interação genótipo $\mathrm{x}$ ambiente (aleatório) e de erros aleatórios; e X, Z e W = matrizes de incidência para b, g e c, respectivamente. As distribuiçôes e estruturas de médias (E) e variâncias (Var) assumidas foram:

$$
E\left[\begin{array}{c}
y \\
g \\
c \\
e
\end{array}\right]=\left[\begin{array}{c}
X b \\
0 \\
0 \\
0
\end{array}\right] ; \quad \operatorname{Var}\left[\begin{array}{l}
g \\
c \\
e
\end{array}\right]=\left[\begin{array}{ccc}
I \sigma_{g}^{2} & 0 & 0 \\
0 & I \sigma_{c}^{2} & 0 \\
0 & 0 & I \sigma_{e}^{2}
\end{array}\right]
$$

$\mathrm{O}$ ajuste do modelo foi obtido a partir das equaçóes de modelo misto:

$$
\left[\begin{array}{ccc}
X^{\prime} X & X^{\prime} Z & X^{\prime} W \\
Z^{\prime} X & Z^{\prime} Z+I \lambda_{1} & Z^{\prime} W \\
W^{\prime} X & W^{\prime} Z & W^{\prime} W+I \lambda_{2}
\end{array}\right] \times\left[\begin{array}{l}
\hat{b} \\
\hat{g} \\
\hat{c}
\end{array}\right]=\left[\begin{array}{c}
X^{\prime} y \\
Z^{\prime} y \\
W^{\prime} y
\end{array}\right],
$$

onde $\lambda_{I}=\frac{\sigma_{e}^{2}}{\sigma_{g}^{2}}=\frac{1-h_{g}^{2}-c^{2}}{h_{g}^{2}}$; em que: $h_{g}^{2}=\frac{\sigma_{g}^{2}}{\sigma_{g}^{2}+\sigma_{c}^{2}+\sigma_{e}^{2}}$ corresponde à herdabilidade individual, no sentido amplo no bloco; $c^{2}=\frac{\sigma_{c}^{2}}{\sigma_{g}^{2}+\sigma_{c}^{2}+\sigma_{e}^{2}}$ corresponde ao coeficiente de determinação dos efeitos da interaçáo genótipo x ambiente; $\sigma_{\mathrm{g}}^{2}$ é a variância genotípica entre os genótipos de feijão-caupi; $\sigma_{c}^{2}$ é variância da interação genótipo $\mathrm{x}$ ambiente; $\sigma_{\mathrm{e}}^{2}$ é a variância residual entre parcelas; $r_{g l o c}=\frac{\sigma_{c}^{2}}{\sigma_{g}^{2}+\sigma_{c}^{2}}=\frac{h_{g}^{2}}{h_{g}^{2}+c^{2}}$ corresponde à correlação genotípica dos genótipos, através dos ambientes.

Os estimadores iterativos dos componentes de variância, por REML, via algoritmo EM, são: $\hat{\sigma}_{e}^{2}=\frac{\left[y^{\prime} y-\hat{b}^{\prime} X^{\prime} y-\hat{g}^{\prime} Z^{\prime} y-\hat{c} W^{\prime} y\right]}{[N-r(x)]}$; $\hat{\sigma}_{g}^{2}=\frac{\left[\hat{g}^{\prime} \hat{g}+\hat{\sigma}_{e}^{2} t r C^{22}\right]}{q} ; \hat{\sigma}_{c}^{2}=\frac{\left[\hat{c}^{\prime} c+\hat{\sigma}_{e}^{2} \operatorname{tr} C^{33}\right]}{s} ;$ em que: $\mathrm{C}^{2} \mathrm{eC}^{3}$ advêm de $C^{-1}=\left[\begin{array}{lll}C_{11} & C_{12} & C_{13} \\ C_{21} & C_{22} & C_{23} \\ C_{31} & C_{32} & C_{33}\end{array}\right]^{-1}=\left[\begin{array}{lll}C^{11} & C^{12} & C^{13} \\ C^{21} & C^{22} & C^{23} \\ C^{31} & C^{32} & C^{33}\end{array}\right]$, sendo

$\mathrm{C}$ a matriz dos coeficientes das equaçóes de modelo misto; tr o operador traço matricial; r(x) o posto da matriz X; N, q, $s=$ número total de dados, número de genótipos e número de combinaçóes genótipo $\mathrm{x}$ ambiente, respectivamente.

Por meio desse modelo foram obtidos os preditores BLUP empíricos (eBLUP ou REML/BLUP) dos valores genotípicos livres da interaçáo, dados por $\hat{\mu}+\hat{g}_{i}$, em que $\hat{\mu}$ é a média de todos os ambientes e $\hat{g}_{i}$ é o efeito genotípico livre da interação genótipo x ambiente. Para cada ambiente j, os valores genotípicos $(\mathrm{Vg})$ são preditos por $\hat{\mu}_{j}+\hat{g}_{i}+(\hat{g} e)_{i j}$, em que $\hat{\mu}_{j}$ é a média do ambiente $\mathrm{j}, \hat{g}_{i}$ é o efeito genotípico do genótipo i, no ambiente j, e $(\hat{g} e)_{i j}$ é o efeito da interação $\mathrm{G} \times \mathrm{E}$ relativo ao genótipo i.

A predição dos valores genotípicos capitalizando a interação média (gem) nos diferentes ambientes é dada por $\hat{\mu}_{j}+\hat{g}_{i}+\hat{g} e_{m}$, sendo calculada por: $\hat{\mu}+\frac{\left(\frac{\hat{\sigma}_{g}^{2}+\hat{\sigma}_{c}^{2}}{n}\right)}{\hat{\sigma}_{g}^{2}} \hat{g}_{i}$, em que $\hat{\mu}$ é a média geral de todos os ambientes; n é o número de ambientes e $\hat{g}_{i}$ é o efeito genotípico do genótipo i.

A seleção conjunta, considerando-se simultaneamente a produtividade, a estabilidade e a adaptabilidade dos genótipos de feijão-caupi é dada pela estatística média harmônica da performance relativa dos valores genotípicos preditos: $M H P R V G_{i}=\frac{n}{\sum_{j=1}^{n} \frac{1}{V g_{i j}}}$, em que: n é o número de locais onde se avaliou o genótipo i, $\mathrm{Vg}_{\mathrm{ij}}$ é o valor genotípico do genótipo i no ambiente j, expresso como proporção da média desse ambiente.

\section{RESULTADOS E DISCUSSÃO}

$\mathrm{O}$ coeficiente de variação experimental $(\mathrm{CV})$ foi de 29,81\% (Tabela 2), sendo este valor similar ao de outros trabalhos conduzidos com a cultura do feijão-caupi (Almeida et al., 2012; Santos et al., 2014a, b). Por se tratar da produtividade de grãos, este valor é esperado, visto que este caráter é significativamente influenciado pelas características edafoclimáticas de cada ambiente, verificadas na tabela 1 . Contrariamente, altos valores de $\mathrm{CV}_{\mathrm{g}}$ sáo indicativos de que fração considerável de QMgenótipos foi extraída do QMtotal. A avaliação conjunta de $\mathrm{CV}_{\mathrm{g}}$ e $\mathrm{CV}_{\mathrm{e}}$ é refletida na estatística $\hat{r}_{g \hat{g}}$ (Resende \& Duarte 2007). A acurácia obtida (82\%) revela boa qualidade experimental e, portanto, segurança e credibilidade na seleção de genótipos superiores quanto ao caráter produtividade de grãos. 
A herdabilidade da média dos genótipos $\left(\hat{h}_{m g}^{2}\right)$ é estimada quando se utilizam médias dos blocos como critério de avaliação e/ou seleção (Resende, 2004). Desta forma, em face dos valores obtidos $(0,68)$, há confiabilidade na seleção dos genótipos de feijão-caupi com base nos valores genotípicos preditos. Ao se estimar a herdabilidade individual no sentido amplo $\left(\hat{h}_{g}^{2}\right)$, considera-se a dispersão genética total, o que é pertinente, pois nesta pesquisa buscamos explorar toda a variância genética entre os genótipos de feijão-caupi. A variância dos efeitos genotípicos $\left(\hat{\sigma}_{g}^{2}\right)$ apresentou magnitude similar à variância dos efeitos da interaçáo $\mathrm{Gx} \mathrm{E}\left(\hat{\sigma}_{c}^{2}\right)$. Isto gerou expressiva variação genotípica $(\mathrm{CV})$, equivalendo a $16,37 \%$ em relação à média geral, e a $18 \%$ da variabilidade fenotípica total, representada pela herdabilidade de parcelas individuais $\left(\hat{h}_{g}^{2}\right)$. Resultados em magnitudes semelhantes foram observados por Maia et al. (2009) e Rosado et al. (2012) ao estimarem parâmetros genéticos via REML/BLUP clones de caju e eucalipto, respectivamente.

Em função do maior ou menor grau de adaptabilidade/ estabilidade genética dos indivíduos, a variância da interação genótipo x ambiente $\left(\hat{\sigma}_{c}^{2}\right)$ pode inflacionar a expressão fenotípica de um caráter (Bastos et al., 2007). Esta medida estima o quanto da variaçáo total está representada pela variação da interação $\mathrm{G} \times \mathrm{E}$. Pequenas magnitudes de $\hat{\sigma}_{c}^{2}$ indicariam que a interação pouco influenciou o valor fenotípico (Maia et al., 2009). Assim, um genótipo com boa produtividade, em um ambiente, tenderia a manter o mesmo patamar em ambientes diversos, uma vez que o genótipo responderia favoravelmente às influências ambientais (elevada correlação entre valores genotípicos, através dos locais), além de possuir elevada previsibilidade frente às variaçōes ambientais. A magnitude de $\hat{\sigma}_{c}^{2}$ foi considerável, equivalendo a $20 \%$ da variabilidade fenotípica total. Este valor, comparativamente a $\hat{h}_{g}^{2}(18 \%)$ é significativamente alto, conduzindo uma correlação fenotípica $\left(\hat{r}_{\text {gloc }}\right)$ mediana através dos ambientes. Isto reflete a importância de se avaliar a adaptabilidade e estabilidade dos genótipos de feijão-caupi, visando à recomendação precisa para os produtores da região de Mato Grosso do Sul.

A medida de um caráter gerado nos ensaios de campo diz respeito ao seu valor fenotípico, constituído do efeito genético, efeito do ambiente e da interação desse genótipo num ambiente específico. Nesse particular, o somatório das variâncias genotípica, residual entre parcelas e da interação resulta na variância fenotípica individual $\left(\hat{\sigma}_{f}^{2}\right)$. Apesar de a dispersão residual entre parcelas representar $61,45 \%$ desse valor, progressos genéticos consideráveis foram conseguidos (Tabela 3). Resultados em magnitudes semelhantes foram observados por Maia et al. (2009) e Rosado et al. (2012), que ao avaliarem os efeitos da interaçáo $\mathrm{G} x \mathrm{E}$ sobre clones de cajueiro e eucalipto, respectivamente, via modelos mistos, constataram que a variância residual entre parcelas representou $66,58 \%$ da variância fenotípica individual.

A correlação genotípica média da performance dos materiais genéticos, através dos ambientes $\left(\hat{r}_{\text {gloc }}\right)$, fornece a confiabilidade de quáo constante será o "ranqueamento" dos genótipos de feijão-caupi ao longo dos ambientes testados. Conforme exposto nas tabelas 2 e 3, houve alteraçóes no ordenamento dos genótipos, em virtude da magnitude mediana da $\hat{r}_{\text {gloc }}(0,48)$ e em função das diferentes magnitudes de correlação genotípica entre os genótipos ao longo dos ambientes (Figura 1). Isto indica a presença da parte complexa da interação $\mathrm{G} \times \mathrm{E}$, representando certa dificuldade na seleção de genótipos de adaptação mais ampla.

Os genótipos BRS Paraguaçu, MNC99-542F-5 e MNC99-508G-1 apresentaram os melhores valores genotípicos para média de todos os ambientes, além de serem selecionados em pelo menos três ambientes (Tabela 3). Os ganhos

Tabela 2. Estimativas dos componentes da variância (REML individual) para a produtividade de grãos de 20 genótipos de feijão-caupi de porte semiprostrado, cultivados em quatro ambientes em Mato Grosso do Sul

\begin{tabular}{|cccc|}
\hline \multicolumn{5}{c}{ Componentes da variância (REML individual) } \\
\hline$\hat{\sigma}_{g}^{2}$ & $9.229,64$ & $\hat{r}_{g \hat{g}}$ & 0,82 \\
$\hat{\sigma}_{c}^{2}$ & $9.960,81$ & $\mathrm{c}^{2}$ & 0,20 \\
\hline$\hat{\sigma}_{e}^{2}$ & $30.589,93$ & $\hat{r}_{g l o c}$ & 0,48 \\
\hline$\hat{\sigma}_{f}^{2}$ & $49.780,37$ & $\mathrm{CV}_{\mathrm{g}}(\%)$ & 16,37 \\
$\hat{h}_{g}^{2}$ & $0,18 \pm 0,07$ & $\mathrm{CV}_{\mathrm{e}}(\%)$ & 29,81 \\
$\hat{h}_{m g}^{2}$ & 0,68 & $\mu\left(\mathrm{kg} \mathrm{ha}^{-1}\right)$ & 586,77 \\
\hline
\end{tabular}

$\hat{\sigma}_{g}^{2}$ : variância genotípica; $\hat{\sigma}_{c}^{2}$ : variância da interaçăo genótipo x ambiente; $\hat{\sigma}_{e}^{2}$ : variância residual entre parcelas; $\hat{\sigma}_{f}^{2}$ : variância fenotípica individual; $\hat{h}_{g}^{2}$ : coeficiente de herdabilidade individual no sentido amplo; $\hat{h}_{m g}^{2}$ : herdabilidade da média do genótipo; $\hat{r}_{g \hat{g}}$ : acurácia na seleçấo dos genótipos; $\mathrm{c}^{2}:$ coeficiente de determinaçāo dos efeitos da interação genótipo x ambiente; $\hat{r}_{\text {gloc }}$ : correlaçâo genotípica através dos ambientes; $\mathrm{CV}$ coeficiente de variaçăo genética; $\mathrm{CV}_{e}$ : coeficiente de variaçăo experimental; $\mu$ : média geral.

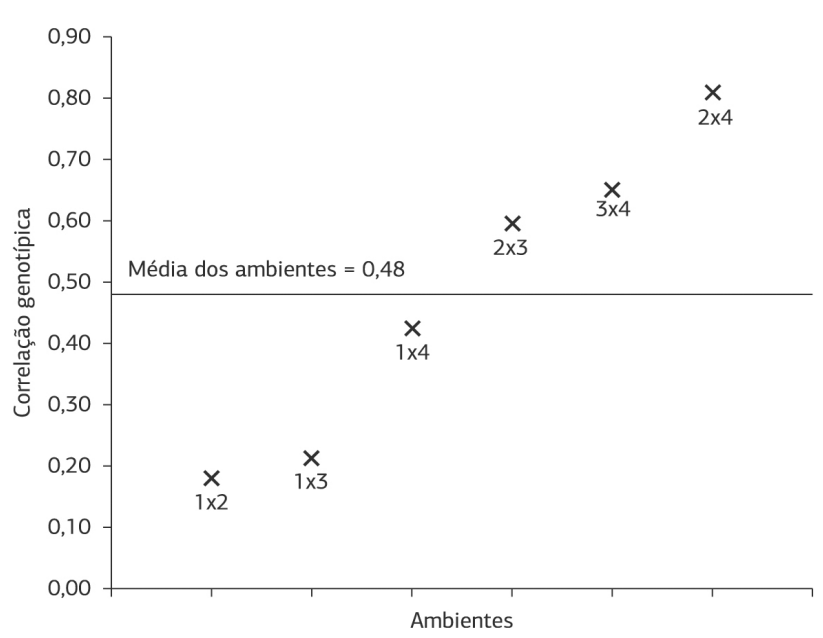

Figura 1. Dispersão dos valores do coeficiente de correlação genotípica obtidos a partir da produtividade de grãos de 20 genótipos de fejião-caupi de porte semiprostado ao longo dos ambientes avaliados. Ambiente 1: Aquidauana (2005); Ambiente 2: Chapadáo do Sul (2005); Ambiente 3: Aquidauana (2006); Ambiente 4: Dourados (2006). 
Tabela 3. Estimativas dos valores genotípicos da produtividade de grãos $\left(\mathrm{kg} \mathrm{ha}^{-1}\right)$ de 20 genótipos de fejião-caupi de porte semiprostado para quatro ambientes e para o ambiente médio

\begin{tabular}{|c|c|c|c|c|c|}
\hline Genótipo & $\begin{array}{l}\text { Ambiente } 1 \\
\hat{\mu}_{1}+\hat{g}+\hat{g} e\end{array}$ & $\begin{array}{l}\text { Ambiente } 2 \\
\hat{\mu}_{2}+\hat{g}+\hat{g} e\end{array}$ & $\begin{array}{l}\text { Ambiente } 3 \\
\hat{\mu}_{3}+\hat{g}+\hat{g} e\end{array}$ & $\begin{array}{l}\text { Ambiente } 4 \\
\hat{\mu}_{4}+\hat{g}+\hat{g} e\end{array}$ & $\begin{array}{c}\text { Ambiente médio } \\
\qquad \hat{\mu}+\hat{g}\end{array}$ \\
\hline MNC99-505G-11 & 423,36 & $1.140,76$ & 539,08 & 378,19 & 495,67 \\
\hline MNC99-507G-4 & 473,26 & $1.149,14$ & 516,94 & 371,76 & 522,06 \\
\hline MNC99-507G-8 & 450,95 & $1.157,50$ & 567,06 & 429,19 & 541,88 \\
\hline TE96-290-12G & 540,40 & $1.164,25$ & 631,43 & 435,46 & 623,08 \\
\hline MNC99-510G-8 & 517,04 & $1.171,11$ & 507,57 & 352,58 & 522,06 \\
\hline MNC99-510F-16 & 486,66 & $1.178,33$ & 613,50 & 397,20 & 560,96 \\
\hline TE97-309G-18 & 393,56 & $1.186,33$ & 470,44 & 342,31 & 417,38 \\
\hline TE97-304G-4 & 508,17 & $1.194,13$ & 527,38 & 415,12 & 590,57 \\
\hline TE97-304G-12 & 590,27 & $1.202,96$ & 665,93 & 442,96 & 688,15 \\
\hline TE97-309G-24 & 441,25 & $1.213,14$ & 598,73 & 452,50 & 630,01 \\
\hline MNC99-508G-1 & 550,09 & $1.221,78$ & 758,85 & 467,19 & 699,86 \\
\hline MNC99-541F-15 & 498,09 & $1.231,14$ & 647,70 & 409,28 & 623,35 \\
\hline MNC99-541F-18 & 557,18 & $1.239,25$ & 686,29 & 402,87 & 664,99 \\
\hline MNC99-541F-21 & 432,52 & $1.246,14$ & 552,01 & 365,17 & 482,83 \\
\hline MNC99-542F-5 & 584,58 & $1.254,93$ & 737,05 & 526,36 & 770,63 \\
\hline MNC99-542F-7 & 415,15 & $1.265,42$ & 581,88 & 384,33 & 553,49 \\
\hline MNC99-547F-2 & 406,32 & $1.281,17$ & 496,54 & 359,23 & 449,03 \\
\hline BRS Paraguaçu & 567,31 & $1.297,49$ & 817,19 & 495,32 & 770,63 \\
\hline BR 17-Gurgueia & 527,62 & $1.312,11$ & 709,87 & 422,13 & 635,70 \\
\hline CNC $\times 409-11 \mathrm{~F}-\mathrm{P} 2$ & 461,86 & $1.340,38$ & 482,90 & 391,37 & 493,04 \\
\hline Média & 491,28 & $1.222,37$ & 539,08 & 412,03 & 586,77 \\
\hline
\end{tabular}

genéticos obtidos com a seleção dos referidos genótipos foram, respectivamente: $18,79 \% ; 18,79 \%$ e $18,04 \%$. Estes valores genotípicos podem, também, ser considerados para recomendaçóes desses genótipos, selecionados em outros ambientes com padrão de interação $\mathrm{G} \times \mathrm{E}$ similar à verificada neste experimento. Segundo Maia et al. (2009), isto ocorre devido à metodologia de modelos mistos que penaliza os valores genotípicos preditos. Assim, o mesmo comportamento das médias genéticas $(\hat{\mu}+\hat{g})$ da produtividade de grãos é esperado, quando os genótipos supracitados forem submetidos a ambientes diversos.

É possível verificar na tabela 4 que o valor genotípico para a média dos ambientes $(\hat{\mu}+\hat{g})$ gerou resultados similares aos métodos em que se capitaliza a adaptabilidade (PRVG) e a adaptabilidade e estabilidade (MHPRVG), simultaneamente. De acordo com Maia et al. (2009), nessa capitalização da interação está intrínseca a escolha dos genótipos mais estáveis e mais adaptados à gama de ambientes ensaiados. Assim, aqui novamente se repetem os três genótipos escolhidos pelo critério de médias genéticas livres da interação $(\hat{\mu}+\hat{g})$ : BRS Paraguaçu, MNC99-542F-5 e MNC99-508G-1. Isto indica que estes genótipos apresentaram maior sinergismo adaptativo em meio aos quatro ambientes analisados, além de possuir boa previsibilidade, isto é, a manutenção da produtividade frente a ambientes diversos. Estes resultados corroboram com Maia et al. (2009) e Regitano et al. (2013), que verificaram manutenção no ordenamento de clones de cajueiro e genótipos de arroz, respectivamente, pelas
Tabela 4. Estabilidade de valores genéticos (MHVG), adaptabilidade de valores genéticos (PRVG e PRVG $\mu$ ), estabilidade e adaptabilidade de valores genéticos (MHPRVG e MHPRVG $\mu$ ) de 20 genótipos de feijāo-caupi semiprostado preditos pela análise BLUP

\begin{tabular}{|c|ccccc|}
\hline Genótipo & MHVG & PRVG & PRVG $\mu$ & MHPRVG & MHPRVG $\mu$ \\
\hline MNC99-505G-11 & 374,22 & 0,81 & 478,16 & 0,81 & 473,66 \\
\hline MNC99-507G-4 & 385,97 & 0,85 & 496,69 & 0,84 & 492,15 \\
\hline MNC99-507G-8 & 434,58 & 0,93 & 545,69 & 0,91 & 535,26 \\
\hline TE96-290-12G & 540,57 & 1,12 & 654,78 & 1,10 & 648,24 \\
\hline MNC99-510G-8 & 388,62 & 0,87 & 512,10 & 0,84 & 493,66 \\
\hline MNC99-510F-16 & 457,86 & 0,96 & 566,04 & 0,96 & 565,64 \\
\hline TE97-309G-18 & 215,41 & 0,58 & 338,54 & 0,50 & 295,39 \\
\hline TE97-304G-4 & 462,18 & 0,99 & 584,81 & 0,98 & 572,99 \\
\hline TE97-304G-12 & 590,40 & 1,23 & 722,85 & 1,21 & 710,76 \\
\hline TE97-309G-24 & 464,31 & 1,03 & 606,47 & 1,00 & 588,60 \\
\hline MNC99-508G-1 & 602,85 & 1,26 & 738,97 & 1,24 & 725,07 \\
\hline MNC99-541F-15 & 502,80 & 1,07 & 625,71 & 1,06 & 624,64 \\
\hline MNC99-541F-18 & 542,66 & 1,16 & 680,73 & 1,14 & 670,41 \\
\hline MNC99-541F-21 & 368,29 & 0,80 & 467,91 & 0,79 & 466,40 \\
\hline MNC99-542F-5 & 683,96 & 1,40 & 822,65 & 1,38 & 810,13 \\
\hline MNC99-542F-7 & 389,30 & 0,88 & 515,55 & 0,86 & 503,54 \\
\hline MNC99-547F-2 & 328,68 & 0,73 & 425,43 & 0,71 & 419,04 \\
\hline BRS Paraguaçu & 661,92 & 1,39 & 815,45 & 1,36 & 795,31 \\
\hline BR 17-Gurgueia & 539,92 & 1,13 & 664,02 & 1,12 & 654,59 \\
\hline CNC x 409-11F-P2 & 353,32 & 0,81 & 472,82 & 0,75 & 442,30 \\
\hline
\end{tabular}

metodologias do valor genotípico para a média dos anos $(\hat{\mu}+\hat{g})$, MHVG, PRVG e MHPRVG, atribuindo tais resultados à correlação genotípica através dos locais $\left(\hat{r}_{\text {gloc }}\right)$, que foi positiva e de magnitude similar a desta pesquisa. 


\section{CONCLUSÃO}

Os genótipos BRS Paraguaçu, MNC99-542F-5 e MNC99-508G-1 podem ser cultivados em vários ambientes no Estado do Mato Grosso do Sul, pois reúnem alta produtividade de grãos, adaptabilidade e estabilidade.

A herdabilidade da média dos genótipos apresenta magnitude variando de moderada a alta, fato que indica excelentes possibilidades para a seleção, permitindo acurácia seletiva de $82 \%$.

Houve concordância entre as estatísticas $\hat{\mu}+\hat{g}$, MHVG, PRVG e MHPRVG na discriminação dos genótipos mais produtivos e com alta adaptabilidade e estabilidade, indicando que elas podem fazer parte de critérios seletivos na rotina dos programas de melhoramento do feijáo-caupi.

\section{REFERÊNCIAS}

Almeida, W. S., Fernandes, F. R. B., Teófilo, E. M., \& Bertini, C. H. C. M. (2012). Adaptability and stability of grain yield in cowpea under different biometrics. Revista Brasileira de Agrociência, 18, 221-228.

Bastos, I. T., Barbosa, M. H. P., Resende, M. D. V., Peternelii, L. A., Silveira, L. C. I., Donda, L. R., Fortunato, A. A., Costa, P. M. A., \& Figueiredo, I. C. R. (2007). Avaliação da interação genótipo x ambiente em cana-de-açúcar via modelos mistos. Pesquisa Agropecuária Tropical, 37, 195-203.

Carbonell, S. A. M., Chioratto, A. F., Resende, M. D. V., Dias, L. A. S., Beraldo, A. L. A., \& Perina, E. F. (2007). Estabilidade de cultivares e linhagens de feijoeiro em diferentes ambientes no Estado de São Paulo. Bragantia, 66, 193-201. http://dx.doi.org/10.1590/S000687052007000200003.

Cruz, C. D., Carneiro, P. C. S., \& Regazzi, A. J. (2014). Modelos biométricos aplicados ao melhoramento genético (3. ed.). Viçosa: Editora UFV. 668 p.
Maia, M. C. C., Resende, M. D. V., Paiva, J. R., Cavalcanti, J. J. V., \& Barros, L. M. (2009). Seleção simultânea para produção, adaptabilidade e estabilidade genotípicas em clones de cajueiro, via modelos misto. Pesquisa Agropecuária Tropical, 39, 43-50.

Regitano, A. No., Ramos, E. A. Jr., Gallo, P. B., Freitas, J. G., \& Azzini, L. E. (2013). Comportamento de genótipos de arroz de terras altas no estado de São Paulo. Revista Ciência Agronômica, 44, 512-519. http://dx.doi.org/10.1590/S1806-66902013000300013.

Resende, M. D. V., Prates, D. F., Yamada, C. K., \& Jesus, A. (1996). Estimação de componentes de variância e predição de valores genéticos pelo método da máxima verossimilhança restrita (REML) e melhor predição linear não viciada (BLUP) em pinus. Boletim de Pesquisa Florestal, 32-33, 23-42.

Resende, M. D. V. (2004). Métodos estatísticos ótimos na análise de experimentos de campo. Colombo: Embrapa Florestas. 57 p.

Resende, M. D. V. (2007). SELEGENREML/BLUP: sistema estatístico e seleção genética computadorizada via modelos lineares mistos. Colombo: Embrapa Florestas. 359 p.

Resende, M. D. V., \& Duarte, J. B. (2007). Precisão e controle de qualidade em experimentos de avaliação de cultivares. Pesquisa Agropecuária Tropical, 37, 182-194.

Rosado, A. M., Rosado, T. B., Alves, A. A., Laviola, B. G., \& Bhering, L. L. (2012). Seleção simultânea de clones de eucalipto de acordo com produtividade, estabilidade e adaptabilidade. Pesquisa Agropecuaria Brasileira, 47, 964-971. http://dx.doi.org/10.1590/ S0100-204X2012000700013.

Santos, J. A. S., Teodoro, P. E., Correa, A. M., Soares, C. M. G., Ribeiro, L. P., \& Abreu, H. K. A. (2014a). Desempenho agronômico e divergência genética entre genótipos de feijão-caupi cultivados no ecótono Cerrado/Pantanal. Bragantia, 73, 377-382. http://dx.doi. org/10.1590/1678-4499.0250.

Santos, J. A. S., Soares, C. M. G., Corrêa, A. M., Teodoro, P. E., Ribeiro, L. P., \& Abreu, H. K. A. (2014b). Agronomic performance and genetic dissimilarity among cowpea (Vigna unguiculata (L.) Walp.) genotypes. Global Advanced Research Journal of Agricultural Science, 3, 271-277. 